\title{
Average Transmission Rate and Outage Performance of Relay-Assisted Satellite Hybrid FSO/RF Systems
}

\author{
Thang V. Nguyen ${ }^{1}$, Hoang D. Le ${ }^{1}$, Ngoc T. Dang ${ }^{1,2}$, and, Anh T. Pham ${ }^{1}$ \\ ${ }^{1}$ Computer Communications Laboratory, the University of Aizu, Aizu Wakamatsu City, Japan \\ ${ }^{2}$ Faculty of Telecommunications, Posts and Telecommunications Institute of Technology, Hanoi, Vietnam \\ Email: \{d8212104, d8202101, pham\}@u-aizu.ac.jp, ngocdt@ptit.edu.vn
}

\begin{abstract}
This paper proposes a rate adaptation design for high altitude platform (HAP)-aided relaying satellite hybrid free-space optical/radio-frequency (FSO/RF) systems. The design allows switching between two links gradually to reduce the frequent link switching in error-prone environments of conventional fixed-rate hybrid FSO/RF systems. System performance metrics, including average transmission rate and outage probability, are analytically studied under the presence of clouds and fading of FSO and RF channels modeled respectively by Gamma-Gamma and Rician distributions. The numerical results quantitatively highlight the effectiveness of our proposed systems over the conventional ones. Moreover, Monte-Carlo simulations are also performed to validate the accuracy of the analytical model.

Index Terms-Satellite communication, adaptive transmission rate, hybrid FSO/RF system
\end{abstract}

\section{INTRODUCTION}

According to the annual disaster statistic report, the damage from flood and storm causes a substantial economic burden and deadliest worldwide, especially in Asia ( $40 \%$ of disaster events [1]). In order to reduce the loss of property and people, it is crucial for Vietnam and other Asian countries to deploy disaster management communications based on the mobile station which used to support search and rescue [2]. However, this solution is difficult to deploy in an inaccessible area. An efficient solution provides uninterrupted communications, high speed, large coverage area, flexible deployment that is optical satellite communications [3]. As a matter of fact, the connection between the satellite and ground station is sensitive to weather conditions such as clouds [4]. To maintain the communications, a high-altitude platform (HAP) is proposed in the middle of satellite-to-ground to avoid cloud coverage as shown in Fig. 1. The complementary of HAP for satellite communications have paved the way for an integrated spaceaerial-ground network [5].

By using HAP as a relay, the dual-hop satellite-HAP-ground is divided into two parts: (i) satellite-to-HAP, (ii) HAP-toground. Since HAP situates above the clouds, the optical beam from satellite to HAP is less sensitive to clouds and atmospheric turbulence. However, the laser link between aerial relay stations like HAP and ground stations is significantly affected by clouds, and atmospheric turbulence [4] [6]. Hence, RF backup link becomes an efficient solution due to less susceptibility to adverse issues of FSO link [3]. Therefore, the hybrid FSO/RF system will be used to counteract the effect of transmission media.

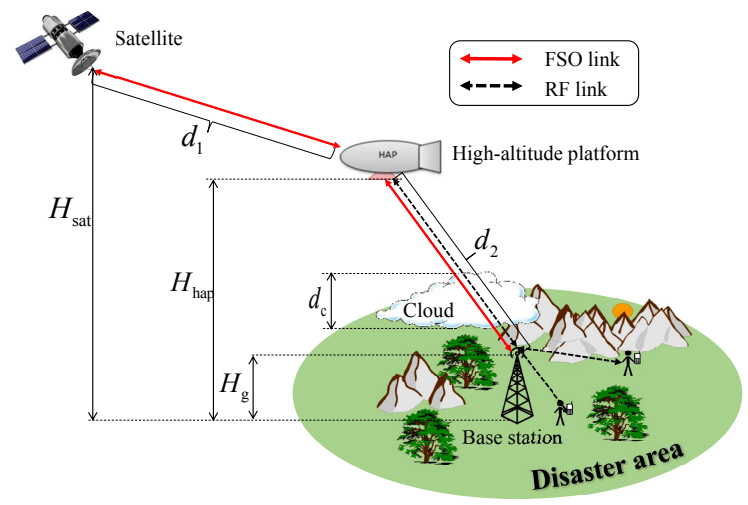

Fig. 1. An example of aerial networks assisted disaster management.

Recently, there have been several studies on the design of dual-hop satellite-HAP-ground systems using hybrid FSO/RF communications [3], [7]. Notably, the authors in [7] investigated the performance of the HAP relay assisted for hybrid FSO/RF downlink system was carry out using Gamma-Gamma distribution to model the FSO channel, and Rician distribution to model the RF channel. In [3], the average symbol error rate and outage performance of the ground-HAP-satellite uplink system were well investigated. The RF channel was modeled by Rician distribution, and FSO channel modeling was taken turbulence-induced fading, beam wander induced pointing error, and free-space loss into account. Aside from [3], [7], to the best of the author's knowledge, there are no other studies on the dual-hop satellite-HAP-ground with the hybrid system in the literature. However, the limitations of these studies were the costly decode-and-forward relaying solution and conventional fixed-rate hybrid FSO/RF system, which frequently switched between FSO and RF links, led to degrading the system performance.

We, therefore, aim at proposing the design of rate adaptation for the dual-hop satellite-HAP-ground downlink systems, which is expected to avoid regularly switching between two links. Rate adaptation means that FSO and RF link can switch to each other, and each link has multiple modes to gradually decrease transmission rate, stay in current mode, or return to higher mode based on the channel state information (CSI). Besides, amplify-and-forward relaying is used in our design to relax the system hardware complexity and cost-effective. 


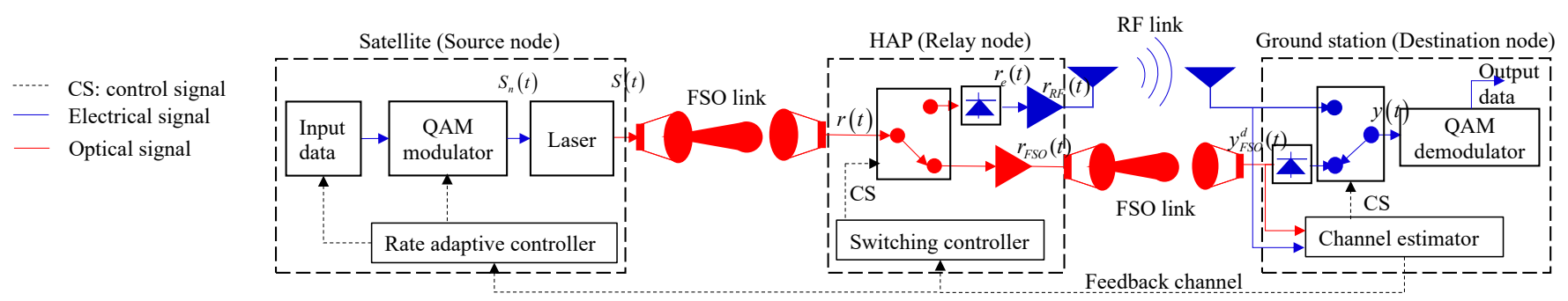

Fig. 2. Dual-hop hybrid FSO-FSO/RF system block diagram.

\section{SySTEM DESCRIPTIONS}

Figure 2 describes a block diagram of dual-hop satelliteHAP-ground with an FSO system at first hop and hybrid FSO/RF system at the second hop. In the considered system, the satellite is a source node. The HAP is a relay node that amplifies the received signal from the satellite then forwards it to the destination. The ground station is a destination node. At the transmitter, the data is modulated by a QAM modulator, and the output signal can be expressed as

$$
s_{n}(t)=A_{n I} g(t) \cos \left(2 \pi f_{r} t\right)-A_{n Q} g(t) \sin \left(2 \pi f_{r} t\right),
$$

where $g(t)$ is the pulse shaping function, $f_{r}$ is the subcarrier frequency, $0 \leq t \leq T$ with $T$ is the interval time of the QAM symbol, $A_{n I}=\{2 n-1-I\}_{n=1}^{I}$ and $A_{n Q}=$ $\{2 n-1-Q\}_{n=1}^{Q}$ are the amplitude of the information signal belong to in-phase and quadrature, respectively. Then, the electrical QAM signal is used to modulate the intensity of optical beam to generate the SC-QAM optical signal, which can be shown as

$$
s(t)=P_{s}\left[1+m s_{n}(t)\right],
$$

where $P_{s}$ is the transmitted power at the satellite, $m$ is the modulation index. At the HAP, i.e., relay node, the optical aperture collects the optical signals then forwards them to an optical switch. Based on channel state information (CSI), the signal is decided to transmit RF or FSO link in the next state. If the RF link is selected, the optical signal is converted to the electrical signal by photodiode then amplify before transmitting to the destination node. If the FSO link is chosen, the optical signal is amplified and directly forward to the ground station. The representative of the output signal of the relay node can be expressed as

\section{For RF link:}

$$
r_{r}(t)=G_{\mathrm{RF}}\left[\eta h_{\mathrm{SR}} P_{s} m s_{n}(t)+\eta n_{\mathrm{b} 1}+n_{\text {re_hap }}\right],
$$

\section{For FSO link:}

$$
r_{f}(t)=G_{\mathrm{F}}\left[h_{\mathrm{SR}} s(t)+n_{\mathrm{b} 1}\right],
$$

where $\eta$ is the optical-to-electrical coefficient, $h_{\mathrm{SR}}$ is the beam spreading loss at the first hop, $n_{\mathrm{b} 1}$ is the background noise, and $n_{\text {re }}$ hap is the receiver noise, $G_{\mathrm{RF}}$ is the electrical amplifier gain, $G_{\mathrm{F}}$ is the optical amplifier gain. Based on CSI, the FSO link will be used if the channel quality is good. Otherwise, the RF link will be applied if the channel condition is poor. The received signal at the ground station can be expressed as

\section{For RF link:}

$$
\begin{aligned}
y_{r}(t) & =h_{\mathrm{RF}} G_{\mathrm{RF}} \eta h_{\mathrm{SR}} P_{s} m s_{n}(t)+h_{\mathrm{RF}} G_{\mathrm{RF}} \eta n_{\mathrm{b} 1} \\
& +h_{\mathrm{RF}} G_{\mathrm{RF}} n_{\text {re_hap }}+n_{\mathrm{re}} .
\end{aligned}
$$

For FSO link:

$$
\begin{aligned}
y_{f}(t) & =h_{\mathrm{FSO}} \eta G_{\mathrm{F}} h_{\mathrm{SR}} P_{s} m s_{n}(t) \\
& +\eta h_{\mathrm{FSO}} G_{\mathrm{F}} n_{\mathrm{b} 1}+\eta n_{\mathrm{b} 2}+n_{\mathrm{rec}}
\end{aligned}
$$

where $h_{\mathrm{RF}}$ and $h_{\mathrm{FSO}}$ are the channel coefficient of the RF link and the FSO link, respectively. $n_{\text {re }}$ and $n_{\text {rec }}$ are receiver noise. By using $G_{\mathrm{RF}}^{2}=\frac{1}{\left(P_{s} m h_{\mathrm{SR}}\right)^{2}+\left(\sigma_{\mathrm{bl}}\right)^{2}}$ and $G_{\mathrm{F}}^{2}=\frac{1}{\left(P_{s} m h_{\mathrm{SR}}\right)^{2}+\left(\sigma_{\mathrm{b} 1}\right)^{2}}$, then, the SNR at the destination node, denoted as $\gamma_{f}^{e 2 e}$ for FSO-FSO link and $\gamma_{r}^{e 2 e}$ for FSO-RF link, can be given as

\section{For FSO-RF link:}

$$
\begin{aligned}
\gamma_{r}^{e 2 e} & =\frac{\left(h_{\mathrm{RF}} G_{\mathrm{RF}} \eta h_{\mathrm{SR}} P_{s} m\right)^{2}}{\left(h_{\mathrm{RF}} G_{\mathrm{RF}} \eta \sigma_{\mathrm{b} 1}\right)^{2}+\left(h_{\mathrm{RF}} G_{\mathrm{RF}} \sigma_{\mathrm{re}_{-} \text {hap }}\right)^{2}+\left(\sigma_{\mathrm{re}}\right)^{2}}, \\
& =\frac{\gamma_{1} \gamma_{2, \mathrm{RF}}}{\gamma_{1}+\gamma_{2, \mathrm{RF}}+1} .
\end{aligned}
$$

\section{For FSO-FSO link:}

$$
\begin{aligned}
\gamma_{f}^{e 2 e} & =\frac{\left(h_{\mathrm{FSO}} \eta G_{\mathrm{F}} h_{\mathrm{SR}} P_{s} m\right)^{2}}{\left(h_{\mathrm{FSO}} \eta G_{\mathrm{F}} \sigma_{\mathrm{b} 1}\right)^{2}+\left(\eta \sigma_{\mathrm{b} 2}\right)^{2}+\left(\sigma_{\mathrm{rec}}\right)^{2}}, \\
& =\frac{\gamma_{1} \gamma_{2, \mathrm{FSO}}}{\gamma_{1}+\gamma_{2, \mathrm{FSO}}+1} .
\end{aligned}
$$

where $\gamma_{1}=\frac{\left(P_{s} m h_{\mathrm{sR}}\right)^{2}}{\left(\sigma_{\mathrm{bl}}\right)^{2}}$ is the SNR of FSO link at the first hop. In the second hop, $\gamma_{2, \mathrm{RF}}=\frac{\left(\eta h_{\mathrm{RF}}\right)^{2}}{\left(h_{\mathrm{RF}} G_{\mathrm{RF}} \sigma_{\mathrm{re}-} \text { hap }\right)^{2}+\left(\sigma_{\mathrm{re}}\right)^{2}}$ is the SNR of the RF link. And, $\gamma_{2, \mathrm{FSO}}=\frac{\left(\eta h_{\mathrm{FS}}\right)^{2}}{\left(\eta \sigma_{\mathrm{b} 2}\right)^{2}+\left(\sigma_{\mathrm{rec}}\right)^{2}}$ is the SNR of FSO link at the second hop.

\section{Channel Modeling}

\section{A. Satellite-to-HAP FSO Link}

As described in the previous section, the effects of clouds and atmospheric turbulence on the optical beam from satellite to HAP can be negligible due to HAP situates above the clouds (i.e., 17-25 km). An optical Gaussian beam is diverged by some amount over the path from the satellite to HAP. The divergence loss is calculated as the fraction of collected power 
of HAP's aperture compare to the area of beam footprint at HAP. For a single beam, the loss can be approximated as

$$
h_{\mathrm{SR}} \approx A_{0} \exp \left(-\frac{2 \rho^{2}}{w_{d e q}^{2}}\right)
$$

where $w_{\mathrm{deq}}^{2}=w_{d}^{2} \frac{\sqrt{\pi} \operatorname{erf}(v)}{2 v \exp \left(-v^{2}\right)}$ is the equivalent beam waist, in which $w_{z}=w_{0} \sqrt{1+\varepsilon\left(\frac{\lambda d_{1}}{\pi \omega_{0}^{2}}\right)^{2}}$ is the beam waist at the distance $d_{1}, w_{0}=(2 \lambda) /(\pi \theta)$ is the beam waist at $d_{1}=0$ with $\theta$ is the divergence angle, $\varepsilon=\left(1+2 \frac{w_{0}^{2}}{\rho_{0}^{2}\left(d_{1}\right)}\right)$, and $\rho_{0}\left(d_{1}\right)=\left(0.55 C_{n}^{2} k_{w}^{2} d_{1}\right)^{-3 / 5}$ is the coherence length [8]. By assuming that active tracking between the satellite and the HAP is perfect, then the detector of HAP is located at the center of beam footprint (i.e., $\rho=0$ ). Hence, $A_{0}=[\operatorname{erf}(v)]^{2}$ corresponds to the fraction of the collected power at $\rho=0$ with $v=\frac{a \sqrt{\pi}}{w_{\mathrm{d}} \sqrt{2}}$ is the ratio between aperture radius and beamwidth.

\section{B. HAP-to-Ground Hybrid FSO/RF Link}

1) RF channel: Two individual FSO and RF links between HAP and the ground station are employed from HAP to the ground station. For the RF link, due to line-of-sight (LOS) transmission between HAP and ground station, the Rician distribution is suitable to model the fading channel. The channel coefficient of the RF link can be given as $h_{\mathrm{RF}}=g_{1} h_{r}$, where $g_{1}$ and $h_{r}$ denote the average power gain and fading gain of the RF link, respectively. The power gain of the RF link can be expressed as

$$
g_{1}(d B)=G_{\mathrm{T}}+G_{\mathrm{R}}-L_{\mathrm{F}}-L_{\mathrm{A}}-L_{\mathrm{c}},
$$

where $G_{\mathrm{T}}$ and $G_{\mathrm{R}}$ are the transmit and receive antenna gains, respectively. $L_{\mathrm{A}}$ denotes the gaseous atmosphere loss, $L_{\mathrm{F}}=$ $20 \log _{10}\left(\frac{4 \pi d_{2}}{f_{r}}\right)$ is the free space loss from the HAP to ground station, in which $f_{r}$ is the frequency of the RF link, $d_{2}$ is the transmission distance between HAP and ground station.

Cloud effects: As recommended by ITU-R [9], a mathematical model based on Rayleigh scattering is suitable for calculating the cloud attenuation on frequencies up to 200 $\mathrm{GHz}$, and it is given by

$$
L_{\mathrm{c}}=d_{\mathrm{c}} \alpha_{1}
$$

where $d_{\mathrm{c}}(\mathrm{km})$ is the considered length of clouds, $\alpha_{1}=$ $K_{\mathrm{c}} M_{\mathrm{c}}$ is the specific attenuation within clouds in which $K_{\mathrm{c}}$ $\left((\mathrm{dB} / \mathrm{km}) /\left(\mathrm{g} / \mathrm{m}^{3}\right)\right)$ is the specific attenuation coefficient, $M_{\mathrm{c}}$ $\left(\mathrm{g} / \mathrm{m}^{3}\right)$ is the cloud liquid water content (CLWC).

Rician fading model: Due to a strong light of sight (LOS) between HAP and ground station, the fading gain, $h_{r}$, is modeled by Rician distribution with the probability density function (PDF) given by [10]. Then, the cumulative distribution function (CDF) of the SNR received at the destination, denoted as $\gamma_{2, \mathrm{RF}}$, is shown as

$$
F_{\gamma_{2, \mathrm{RF}}}\left(\gamma_{2, \mathrm{RF}}\right)=1-Q_{1}\left(\sqrt{2 K}, \sqrt{\frac{2(K+1)}{\bar{\gamma}_{2, \mathrm{RF}}} \gamma_{2, R F}}\right)
$$

where $K$ is Rician factor, $Q_{1}(\cdot, \cdot)$ is the Marcum $Q_{1}$-function [10], and $\bar{\gamma}_{2, \mathrm{RF}}$ is the average SNR of the RF link.

2) FSO channel: For an optical link from HAP to ground station, The mathematical model of channel coefficient is expressed as $h_{\mathrm{FSO}}=h_{\mathrm{s}} h_{\mathrm{c}} h_{\mathrm{a}}$, in which $h_{\mathrm{s}}$ is the beam spreading loss, $h_{\mathrm{a}}$ is turbulence-induced fading, and $h_{\mathrm{c}}$ is cloud attenuation. The beam spreading loss is similarly considered as the previous section since the Gaussian beam is used from HAP to the ground station.

Cloud effects: The critical effect of clouds on the FSO link is severe, as recently reported in [4], [11]. In the presence of cloud coverage, system performance and availability can be severely degraded due to high CLWC. Following the BeerLambert law, the cloud attenuation can be expressed

$$
h_{\mathrm{c}}=\exp \left(-\alpha_{2} d_{\mathrm{c}}\right)
$$

where $\alpha_{2}$ is the attenuation coefficient and can be expressed as

$$
\alpha_{2}=\frac{3.91}{V[\mathrm{~km}]}\left(\frac{\lambda[\mathrm{nm}]}{550}\right)^{-q},
$$

where $q$ is the coefficient, which is given by Kim's model as

$$
q= \begin{cases}1.6, & \text { if } V>50 \mathrm{~km}, \\ 1.3, & \text { if } 6 \mathrm{~km}<V \leq 50 \mathrm{~km}, \\ 0.16 V+0.34, & \text { if } 1 \mathrm{~km}<V \leq 6 \mathrm{~km}, \\ V-0.5, & \text { if } 0.5 \mathrm{~km}<V \leq 1 \mathrm{~km}, \\ 0, & \text { if } V \leq 0.5 \mathrm{~km} .\end{cases}
$$

The visibility can be determined based on cloud droplet number concentration $N_{\mathrm{c}}$ and cloud liquid water content $M_{\mathrm{c}}$ as followed

$$
V=\frac{1.002}{\left(N_{\mathrm{c}} \times M_{\mathrm{c}}\right)^{0.6473}} .
$$

The typical values of $N_{\mathrm{c}}$ and $M_{\mathrm{c}}$ for multiple types of clouds are presented in [6].

Turbulence-induced fading: The Gamma-Gamma distribution is used to model the atmospheric turbulence caused by the in-homogeneity in temperature and pressure of the atmosphere along the transmission path from HAP to the ground station. In the FSO system, the Rytov variance is used to measure the strength of atmospheric turbulence and expressed as

$$
\sigma_{R}^{2}=2.25 k_{w}^{7 / 6} \sec ^{11 / 6}(\zeta) \int_{H_{\mathrm{g}}}^{H_{\text {hap }}} C_{n}^{2}(h)\left(h-H_{\mathrm{g}}\right)^{5 / 6} d h,
$$

where $k_{w}=2 \pi / \lambda$ is the optical wave number, $\zeta$ is the zenith angle. In [12], based on Hufnagel-Valley model, the variation of refractive index structure parameter $C_{n}^{2}(h)$ according to the altitude $h$ can be written as

$$
\begin{aligned}
& C_{n}^{2}(h)=0.00594\left(\frac{v_{\text {wind }}}{27}\right)^{2}\left(10^{-5} h\right) \exp \left(-\frac{h}{1000}\right) \\
& +2.7 \times 10^{-16} \exp \left(\frac{-h}{1500}\right)+C_{n}^{2}(0) \exp \left(\frac{-h}{100}\right),
\end{aligned}
$$


TABLE I

AN ILLUSTRATIVE EXAMPLE OF RATE ADAPTATION WITH $M$-QAM

\begin{tabular}{|c|c|c|c|c|c|c|c|c|c|}
\hline \multirow{2}{*}{\multicolumn{2}{|c|}{$\begin{array}{l}\text { Phase } \\
\text { Mode }\end{array}$}} & \multicolumn{5}{|c|}{ FSO } & \multicolumn{2}{|c|}{$\mathrm{RF}$} & Outage \\
\hline & & 1 & 2 & 3 & 4 & 5 & 6 & 7 & 8 \\
\hline \multicolumn{2}{|c|}{ Transmission Rate } & $8 R_{f}$ & $7 R_{f}$ & $6 R_{f}$ & $5 R_{f}$ & $4 R_{f}$ & $5 R_{r}$ & $4 R_{r}$ & 0 \\
\hline \multirow{2}{*}{$\begin{array}{l}\text { SNR } \\
\text { conditions }\end{array}$} & FSO & {$\left[\gamma_{f 5}, \infty\right)$} & {$\left[\gamma_{f 4}, \gamma_{f 5}\right)$} & {$\left[\gamma_{f 3}, \gamma_{f 4}\right)$} & {$\left[\gamma_{f 2}, \gamma_{f 3}\right)$} & {$\left[\gamma_{f 2}, \gamma_{f 1}\right)$} & & {$\left[0, \gamma_{f}\right]$} & \\
\hline & $\mathrm{RF}$ & \multicolumn{5}{|c|}{ No consideration } & {$\left[\gamma_{r 2}, \infty\right.$} & {$\left[\gamma_{r 1}, \gamma_{r 2}\right)$} & {$\left[0, \gamma_{r 1}\right)$} \\
\hline Modulatio & & 256-QAM & 128-QAM & 64-QAM & 32-QAM & 16-QAM & 32-QAM & 16-QAM & No transmission \\
\hline
\end{tabular}

where $C_{n}^{2}(0)$ is the ground turbulence level varying in the range of $10^{-17} \mathrm{~m}^{-2 / 3}$ to $10^{-13} \mathrm{~m}^{-2 / 3}$, and $v_{\text {wind }}(\mathrm{m} / \mathrm{s})$ is the root mean squared wind speed and has a typical value of 21 $\mathrm{m} / \mathrm{s}$.

After transformation of a random variable $h_{\mathrm{FSO}}$, the $\mathrm{CDF}$ of the instantaneous SNR received at the destination via FSO link, denoted as $\gamma_{2, \text { FsO }}$, can be shown as

$$
F_{\gamma_{2, \mathrm{FSO}}}\left(\gamma_{2, \mathrm{FSO}}\right)=\frac{1}{\Gamma(\alpha) \Gamma(\beta)} G_{1,3}^{2,1}\left[\alpha \beta \sqrt{\frac{\gamma_{2, \mathrm{FSO}}}{\bar{\gamma}_{2, \mathrm{FSO}}}} \mid \begin{array}{c}
1 \\
\alpha, \beta, 0
\end{array}\right],
$$

where $\Gamma(\cdot)$ is the gamma function, and $G_{\mathrm{p}, \mathrm{q}}^{\mathrm{m}, \mathrm{n}}(\cdot)$ is MeijerG function. $\alpha$ and $\beta$ are the small scale and large scale of turbulent eddies [12].

\section{Rate AdAPtation Design}

This section focuses on the design of rate adaptation for our considered system. Several performance metrics are, then, analytically derived.

\section{A. Rate Adaptation Operation}

As shown in Fig. 2, the channel estimator is implemented at the destination to estimate the fading on both FSO and RF channel by reflecting the instantaneous end-to-end SNR, which is expressed in Eq. (7) and Eq. (8). Assuming a perfect logical channel for feedback channel state information (CSI), which includes information regarding the selected link and selected transmission rate. Based on the CSI, the switching controller of HAP will decide the transmission link. The FSO link will be used as long as its SNR higher than a certain threshold. In other respect, the RF link will be used until the FSO channel gets better or an outage happens. On the other hand, the rateadaptive controller of the source node will be dynamically adapt transmission rate depending on the CSI.

Table I briefly illustrates the process of rate adaptation in our work. There are three phases: FSO, RF, and outage, in which the FSO phase and RF phase have several modes corresponding to the different transmission rates. In the beginning, the system operates in the highest mode of the FSO phase due to the target of transmitting high speed. Then, the transmission rate is dynamically adapt based on CSI, and if the channel quality does not change or degrades, the system will stay in current mode or move to lower rate mode, respectively. At the lower rate mode, the transmission rate will return to the higher mode if channel quality becomes better. At the lowest rate mode of the FSO phase, the channel quality decreases, leading to the SNR of the FSO link not meeting the requirement; then, the RF link will be used. RF phase has several modes, and its operating mechanism is similar to the FSO phase. At the lowest rate mode of the RF phase, the quality of the RF link gets worse, and the outage will occur (i.e., stop transmitting the data).

To design an adaptive rate for the FSO link and RF link, adaptive modulation is employed for the sake of simplicity. Besides, the SC-QAM is utilized to support highspeed transmission. As a result, when the link is nominated, the modulation order and symbol rate will be chosen based on the instantaneous SNR of the FSO link and RF link. Corresponding to multiple modes of FSO link and RF link, several SNR regions are designed to be compatible with the transmission rate of FSO and RF link according to the rule as followed

$$
R_{k}=\left\{\begin{array}{lll}
R_{f} \log _{2} M & \text { if } \gamma_{f(j)} \leq \gamma_{f}^{e 2 e}<\gamma_{f(j+1)} & \text { FSO link, } \\
R_{r} \log _{2} M & \text { if } \gamma_{r(i)} \leq \gamma_{r}^{e 2 e}<\gamma_{r(i+1)} & \text { RF link, } \\
0 & \text { if } 0 \leq \gamma_{r}<\gamma_{r(1)} & \text { Outage, }
\end{array}\right.
$$

where $R_{f}$ and $R_{r}$ are the symbol rate of the FSO link and RF link, respectively, note that transmission rate depends on the modulation order $M\left(M=2^{t}\right.$ with $t$ is the number of bits per symbol). An example of rate adaptation with $M$-QAM including five modes for FSO link, two modes for RF link, and outage mode is depicted in Table I. How to divide multiple regions? These are implemented to satisfy a requirement of quality of service (QoS). In the physical layer, a target bit error rate $\left(\mathrm{BER}_{0}\right)$ is considered as a usual metric of QoS for both links. By applying SC-QAM, the BER B $_{0}$ over the AWGN channel can be well approximated in [13] and expressed as

$$
\operatorname{BER}(M, \gamma) \cong 0.2 \exp \left[-\frac{3 \gamma}{2(M-1)}\right] .
$$

The switching threshold is derived as

$$
\gamma=\frac{-2(M-1)}{3} \ln [5 \operatorname{BER}(M, \gamma)] .
$$

\section{B. Performance Analysis}

1) Outage Probability: The outage performance is defined as the probability that no data transmission can avoid the high error rate in both FSO and RF modes. As a result, it is computed as

$$
\begin{aligned}
P_{\text {out }} & =\int_{0}^{\gamma_{f(1)}^{e 2 e}} f_{\gamma_{f}}\left(\gamma_{f}^{e 2 e}\right) d \gamma_{f}^{e 2 e} \int_{0}^{\gamma_{r(1)}^{e 2 e}} f_{\gamma_{r}^{e 2 e}}\left(\gamma_{r}^{e 2 e}\right) d \gamma_{r}^{e 2 e}, \\
& =F_{\gamma_{f}^{e 2 e}}\left(\gamma_{f}^{e 2 e}\right) F_{\gamma_{r}^{e 2 e}}\left(\gamma_{r}^{e 2 e}\right),
\end{aligned}
$$



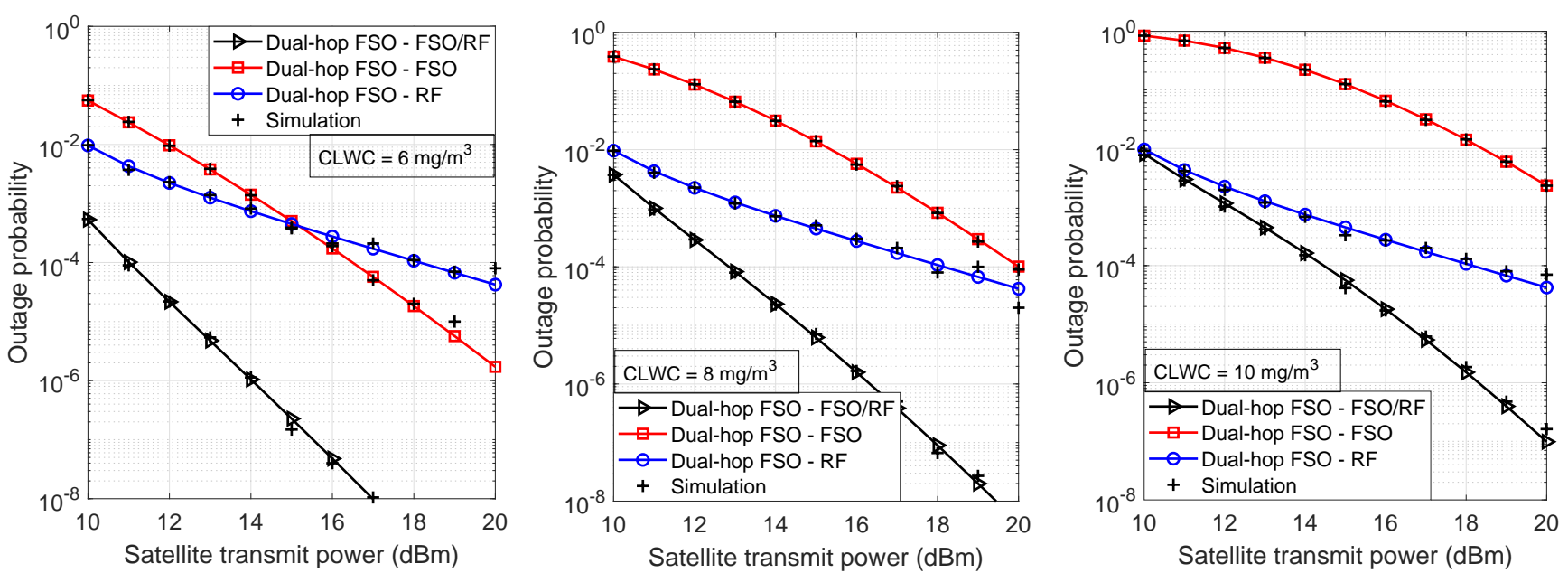

Fig. 3. Outage probability versus satellite's transmitted power for different cloud conditions.

where $F_{\gamma_{r}^{e 2 e}}\left(\gamma_{r}^{e 2 e}\right)$ and $F_{\gamma_{f}^{e 2 e}}\left(\gamma_{f}^{e 2 e}\right)$ are respectively the CDFs of end-to-end SNR for the dual-hop of FSO-RF and FSO-FSO, which is given as

\section{For FSO-RF link:}

$F_{\gamma_{r}^{e 2 e}}\left(\gamma_{r}^{e 2 e}\right)=\operatorname{Pr}\left(\frac{\gamma_{1} \gamma_{2, \mathrm{RF}}}{\gamma_{2, \mathrm{RF}}+1}<\gamma_{r}^{e 2 e}\right)=F_{\gamma_{2, \mathrm{RF}}}\left(\frac{\gamma_{r}^{e 2 e}}{\gamma_{1}-\gamma_{r}^{e 2 e}}\right)$.

\section{For FSO-FSO link:}

$F_{\gamma_{f}^{e 2 e}}\left(\gamma_{f}^{e 2 e}\right)=\operatorname{Pr}\left(\frac{\gamma_{1} \gamma_{2, \mathrm{FSO}}}{\gamma_{2, \mathrm{FSO}}+1}<\gamma_{f}^{e 2 e}\right)=F_{\gamma_{2, \mathrm{FSO}}}\left(\frac{\gamma_{f}^{e 2 e}}{\gamma_{1}-\gamma_{f}^{e 2 e}}\right)$.

2) Average Transmission Rate: The average transmission rate in the proposed system, which is determined through transmission modes of both FSO and RF links, is calculated as

$$
\bar{R}=\sum_{k=1}^{N} p_{k} R_{k}
$$

where $N$ is the total transmission modes, and $R_{k}$ is the corresponding transmission bit rate at the $k$-th mode. $p_{k}$ is the probability of selecting the $k$-th mode, which is written as

\section{For FSO-RF link:}

$$
\begin{aligned}
& p_{k}^{\mathrm{RF}}=\int_{0}^{\gamma_{f(1)}^{e 2 e}} f_{\gamma_{f}^{e 2 e}}\left(\gamma_{f}^{e 2 e}\right) d \gamma_{f}^{e 2 e} \int_{\gamma_{r(i)}^{e 2 e}}^{\gamma_{r(i+1)}^{e 2 e}} f_{\gamma_{r}^{e 2 e}}\left(\gamma_{r}^{e 2 e}\right) d \gamma_{r}^{e 2 e} \\
& =\left[1-F_{\gamma_{f}^{e 2 e}}\left(\gamma_{f(1)}^{e 2 e}\right)\right]\left[F_{\gamma_{r}^{e 2 e}}\left(\gamma_{r(i+1)}^{e 2 e}\right)-F_{\gamma_{r}^{e 2 e}}\left(\gamma_{r(i)}^{e 2 e}\right)\right] .
\end{aligned}
$$

\section{For FSO-FSO link:}

$$
\begin{aligned}
p_{k}^{\mathrm{FSO}} & =\int_{\gamma_{f(j)}^{e 2 e}}^{\gamma_{f(j+1)}^{e 2 e}} f_{\gamma_{f(j)}^{e 2 e}}\left(\gamma_{f(j)}^{e 2 e}\right) d \gamma_{f(j)}^{e 2 e} \\
& =F_{\gamma_{f(j)}^{e 2 e}}\left(\gamma_{f(j+1)}^{e 2 e}\right)-F_{\gamma_{f(j)}^{e 2 e}}\left(\gamma_{f(j)}^{e 2 e}\right)
\end{aligned}
$$

\begin{tabular}{|c|c|c|}
\hline Name & Symbol & Value \\
\hline \multicolumn{3}{|c|}{ LEO satellite } \\
\hline LEO satellite altitude & $H_{\text {sat }}$ & $530 \mathrm{~km}$ \\
\hline Divergence angle & $\theta_{\text {sat }}$ & $15 \mu \mathrm{rad}$ \\
\hline Optical wavelength & $\lambda$ & $1550 \mathrm{~nm}$ \\
\hline RF frequency & $f_{r}$ & $2 \mathrm{GHz}$ \\
\hline \multicolumn{3}{|c|}{ High Altitude Platform (HAP) } \\
\hline HAP altitude & $h_{\text {hap }}$ & $20 \mathrm{~km}$ \\
\hline HAP aperture radius & $r_{\text {hap }}$ & $10 \mathrm{~cm}$ \\
\hline Zenith angle & $\zeta$ & $60^{\circ}$ \\
\hline Divergence angle & $\theta_{\mathrm{HAP}}$ & $1.5 \mathrm{mrad}$ \\
\hline Background light power & $P_{\mathrm{b} 1}$ & $250 \mu W$ \\
\hline \multicolumn{3}{|c|}{ Ground Station (GS) } \\
\hline GS altitude & $H_{\mathrm{g}}$ & $10 \mathrm{~m}$ \\
\hline GS aperture radius & $r_{\mathrm{g}}^{\circ}$ & $10 \mathrm{~cm}$ \\
\hline Detector responsibility & $\mathcal{R}$ & 0.9 \\
\hline Background light power & $P_{\mathrm{b} 2}$ & $10 \mu W$ \\
\hline Gaseous atmosphere losses & $L_{\mathrm{A}}$ & $5.4 \times 10^{-3} \mathrm{~dB} / \mathrm{km}$ \\
\hline Receiver noise bandwidth & $B W$ & $74.8 \mathrm{dBHz}$ \\
\hline System noise temperature & $T_{N}$ & $17.6 \mathrm{dBK}$ \\
\hline Noise figure & $N_{F}$ & $3 \mathrm{~dB}$ \\
\hline \multicolumn{3}{|c|}{ Other parameters } \\
\hline Target BER & $\mathrm{BER}_{0}$ & $10^{-6}$ \\
\hline Number of concentration & $N_{c}$ & $250 \mathrm{~cm}^{-3}$ \\
\hline Considered length of clouds & $d_{c}$ & $2 \mathrm{~km}$ \\
\hline Turbulence at ground level & $C_{n}^{2}(0)$ & $10^{-13} \mathrm{~m}^{-2 / 3}$ \\
\hline Rician factor & $K$ & $6 \mathrm{~dB}$ \\
\hline FSO symbol rate & $R_{f}$ & 400 Msps \\
\hline RF symbol rate & $R_{r}$ & $100 \mathrm{Msps}$ \\
\hline
\end{tabular}

TABLE II

SIMULATION PARAMETERS

\section{Numerical Results}

In this section, we present the numerical results for the performance evaluation of our proposed satellite-HAP-ground system. For the rate adaptation design, five transmission modes are used for the FSO link, including 16, 32, 64, 128, 256QAM. In addition, RF one covers two modes using 16, 32QAM. The system parameters, unless otherwise noted, are given in Table II. Moreover, the Monte Carlo simulations are also performed to validate the accuracy of the proposed 
analytical model.

Firstly, Fig. 3 quantitatively highlights the proposed dualhop FSO-FSO/RF system by comparing the outage performance with that of FSO-FSO and FSO-RF ones over a range of satellite's transmitted power. Also, several cloud conditions are investigated, i.e., CLWC $=6 \mathrm{mg} / \mathrm{m}^{3}, \mathrm{CLWC}=8 \mathrm{mg} / \mathrm{m}^{3}$, and CLWC $=10 \mathrm{mg} / \mathrm{m}^{3}$. As illustrated in the figure, the outage performance of dual-hop FSO-FSO remarkably rises when the amount of CLWC grows while the dual-hop FSO-RF ones do not change. However, if the satellite provides high power enough, the influence of clouds on the dual-hop FSO-FSO system can be reduced. For example, in the most left figure, the dual-hop FSO-RF system performance is greater than the dual-hop FSO-FSO ones over the range of low transmit power (e.g., from 10 to $15 \mathrm{dBm}$ ). In contrast, the dual-hop FSOFSO system outperforms the dual-hop FSO-RF system if the transmit power is high enough (e.g., larger than $16 \mathrm{dBm}$ ). The reason behind is the cloud effect on the optical link is severe due to the Mie scattering phenomenon (i.e., the cloud droplet size is much larger than optical wavelength [4]). Therefore, the more increasing CLWC is, the more significant attenuation FSO suffers. In [9], ITU-R P-840 shows that the attenuation of clouds on the frequencies of the order of $100 \mathrm{GHz}$ is significant. In our proposed system, the frequency for RF link is $2 \mathrm{GHz}$, then the impact of clouds on RF link is small due to the RF wavelength more enormous than the cloud droplet size. By inheriting both FSO and RF advantages, the dual-hop FSOFSO/RF has better performance due to applying link switching and rate adaptation to combat the effect of clouds.

Next, we further highlight the effectiveness of our proposed FSO-hybrid FSO/RF system with the rate adaption design over the conventional one, i.e., fixed-rate transmission. Notably, Fig. 4 shows the impact of clouds on the average transmission rate performance, when the satellite's transmitted power $P_{\mathrm{s}}$ $=13 \mathrm{dBm}$. Additionally, regarding the fixed-rate design, we consider 16, 32, 64, 128, 256-QAM for FSO mode together with 32-QAM for RF mode. As is evident, our proposed design offers considerably better performance compared to conventional design. For example, as CLWC $=8.5 \mathrm{mg} / \mathrm{m}^{3}$, our proposed system is able to achieve 2 Gbps of transmission rate, while the conventional system can only retain the transmission rate of $1.58 \mathrm{Gbps}, 1.47 \mathrm{Gbps}, 1.38 \mathrm{Gbps}, 0.89 \mathrm{Gbps}$, and 0.52 Gbps for 32, 16, 64, 128, 256-QAM FSO, respectively.

\section{CONCLUSIONS}

We proposed to design an adaptive rate scheme for hybrid $\mathrm{FSO} / \mathrm{RF}$ systems in the scenarios of satellite-HAP-ground networks. Weather effects were considered on both links. The Gamma-Gamma distribution is used to model the atmospheric turbulence, while the Rician distribution is used to model the RF channel. Numerical performance metrics such as average transmission rate, outage probability were analytically studied and confirmed accuracy by Monte-Carlo simulation. Our system design quantitatively shows how the dual-hop FSO-FSO/RF system outperforms other systems (e.g., dualhop FSO-FSO system, dual-hop FSO-RF system) under the

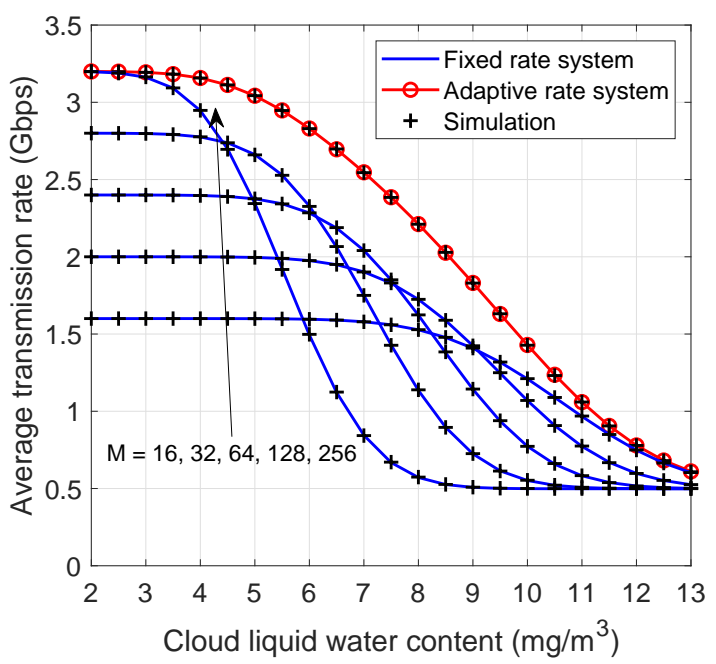

Fig. 4. Impact of clouds to the average transmission rate with the satellite transmit power of $13 \mathrm{dBm}$.

weather effects such as clouds. Moreover, our design of an adaptive rate system provides substantially better performance compared to that of conventional fixed-rate design.

\section{REFERENCES}

[1] "Natural disasters 2019: Now is the time to not give up." Centre for Research on the Epidemiology of Disaster (CRED), Brussels,, 2020.

[2] T. Tokuyasu, N. Tachikawa, S. Uwano, T. Tatsuta, and H. Nakamura, "Wireless access system for disaster recovery providing safety and security to customers," NTT Technical Review, vol. 16, no. 1, 2018.

[3] S. R., S. Sharma, N. Vishwakarma, and A. Madhukumar, "Hapsbased relaying for integrated space-air-ground networks with hybrid fso/rf communication : A performance analysis," IEEE Transactions on Aerospace and Electronic Systems, pp. 1-1, 2021.

[4] L. D. Hoang, T. Nguyen, and A. Pham, "Cloud attenuation statistical model for satellite-based fso communications," IEEE Antennas and Wireless Propagation Letters, pp. 1-1, 2021.

[5] N. Saeed, H. Almorad, H. Dahrouj, T. Y. Al-Naffouri, J. S. Shamma, and M.-S. Alouini, "Point-to-point communication in integrated satelliteaerial networks: State-of-the-art and future challenges," 2020.

[6] E. Erdogan, I. Altunbas, G. K. Kurt, M. Bellemare, G. Lamontagne, and H. Yanikomeroglu, "Site diversity in downlink optical satellite networks through ground station selection," IEEE Access, vol. 9, pp. 3117931190, 2021.

[7] R. Swaminathan, S. Sharma, and A. S. MadhuKumar, "Performance analysis of haps-based relaying for hybrid fso/rf downlink satellite communication," in 2020 IEEE 91st Vehicular Technology Conference (VTC2020-Spring), pp. 1-5, 2020.

[8] F. Yang, J. Cheng, and T. A. Tsiftsis, "Free-space optical communication with nonzero boresight pointing errors," IEEE Transactions on Communications, vol. 62, no. 2, pp. 713-725, 2014

[9] "Attenuation due to clouds and fog." document Rec. ITU-R P-840-6, 2013.

[10] M. Simon and M.-S. Alouini, Digital Communication over Fading Channels, 2nd Edition. Wiley Publishers, 2005.

[11] T. V. Nguyen, H. D. Le, T. V. Pham, and A. T. Pham, "Link availability of satellite-based fso communications in the presence of clouds and turbulence," IEICE Communications Express, vol. 10, 2021.

[12] H. Kaushal and G. Kaddoum, "Optical communication in space: Challenges and mitigation techniques," IEEE Communications Surveys Tutorials, vol. 19, no. 1, pp. 57-96, 2017.

[13] V. V. Mai and A. T. Pham, "Cross-layer designs and analysis of adaptiverate transmission and arq for free-space optical communications," IEEE Photonics Journal, vol. 8, no. 1, pp. 1-15, 2016. 